prevents HIV, STIs, and other STI-related sequelae, including infertility. Furthermore, when couples attempt pregnancy, the unique characteristics of the condom allow it to be used in conjunction with safer conception interventions during the fertile period of the menstrual cycle. The condom may be used with the following: timed intercourse and self-vaginal insemination with or without pre-exposure prophylaxis. Therefore, HIVaffected couples with an immediate desire for a child may use the condom as an MPT, and during the fertile period, the couples may choose either a clinical or pharmacological method in their attempts to achieve pregnancy. Innovative MPTs need to combine methods and technologies in order to assist HIVaffected couples in maintaining their reproductive health, to prevent HIV/STI transmission, and to achieve their reproductive goals of having healthy children when desired. The traditional view of the condom serving only as dual prevention of pregnancy and HIV/STIs needs to be reconsidered, and instead expanded to include its ability to address all disease prevention and reproductive needs of HIV-affected couples. Future designs for MPT methodologies that do encompass the multiple aspects of HIV/STI prevention and reproductive needs may impact MPT demand, uptake, acceptability, and adherence.

\section{S15.4 THE UNMET NEED AND POTENTIAL APPLICATION OF MPTS FOR RECTAL USE: IMPLICATIONS FOR WOMEN AND MSM AT RISK OF STIS AND HIV}

1,2Bridget Haire*. ${ }^{1}$ The Kirby Institute; ${ }^{2}$ Australian Federation of AIDS Organisations

10.1136/sextrans-2015-052270.70

Introduction Both men and women and transgendered people practice receptive anal sex, and an act of anal intercourse is 1020 times more likely to transmit HIV than an act of vaginal intercourse. Condoms are effective at preventing HIV acquisition through receptive anal sex, but many people do not use them perfectly for every sex act. Oral HIV pre-exposure prophylaxis is also effective at preventing HIV acquisition through receptive anal sex, but it does not prevent other sexually transmissible infections. Hence there is a need to develop a wider range of tools so that there are options for optimal protection of sexual health for men and women practicing receptive anal intercourse. Methods This paper reviews the pipeline of products in development that are designed to protect the sexual health of people practicing receptive anal intercourse.

Results A range of products are in development which are designed the rectal use. These products are 'multipurpose' technologies primarily as they aim to combine products frequently used prior to or during anal intercourse for ease and pleasure such as sexual lubricants, and douche and enema products - for HIV and potentially sexually transmissible infection (STI) prevention purposes. Other products include suppositories and specifically designed applicators for rectal use. Currently research is focused on antiretroviral-based HIV prevention technologies, but there has also been some research on non-antiretroviral-based products.In addition, there are developments in oral pre-exposure prophylaxis for STIs other than HIV.

Conclusion Given that adherence to interventions is critical to the current HIV prevention tools, building preventative agents into products that people choose to use to enhance the experience of sex may help to optimize adherence.

Disclosure of interest statement No interests to disclose.

\section{S16 - STI and HIV in pregnancy}

\section{S16.1 ELIMINATING MOTHER TO CHILD TRANSMISSION OF HIV AND SYPHILIS}

Nathalie Broutet* World Health Organization, Geneva, Switzerland

\subsection{6/sextrans-2015-052270.71}

The Initiative for Global Elimination of Congenital Syphilis was launched in 2007, and the Global Plan towards the Elimination of New HIV Infections among Children by 2015 and Keeping Their Mothers Alive was launched in 2010. In 2014, the World Health Organization, jointly with UNAIDS, UNICEF, UNFPA, and other global partners released guidance on the processes and criteria for dual elimination of mother-to-child transmission (MTCT) of HIV and syphilis. Countries all over the world are now striving for dual elimination. This presentation will address recent successes like Cuba and current strategies and new tools that can help to overcome barriers in the dual elimination of MTCT HIV and syphilis with a particular focus on MTCT of syphilis.

\section{S16.2 POTENTIAL FOR UPTAKE OF DIAGNOSTIC TESTING SERVICES ALONG THE CONTINUUM OF CARE: LANDSCAPE ASSESSMENT OF COMMUNITY AND PROVIDERS}

Sadaf Khan*, Matt Steele, Lynn Atuyambe, Peter Waiswa, Nabeel Ashraf Ali, Dunia Faulx, Shamsul Arifeen. PATH, Seattle, USA

\subsection{6/sextrans-2015-052270.72}

Introduction Maternal infections continue to be a major contributor to maternal, perinatal, and neonatal morbidity and mortality. The use of diagnostic technologies for maternal infections in the developing world is poorly researched. The current study aimed to elucidate the provider- and patient-level factors that may act to inhibit or encourage timely and effective provision of diagnostic technologies during the pregnancy, intrapartum, and early postpartum periods for infections linked with poor outcomes for mothers and infants in the developing world.

Methodology This was an exploratory study that employed a socio-ecological framework. Using qualitative data collection methods, including focus group discussions (FGDs) with pregnant women, new mothers and their families, and in-depth interviews (IDIs) with lower-level providers, we examined healthseeking behaviours and perceptions of diagnostic testing during pregnancy with the aim of obtaining an in-depth understanding of factors related to the potential for uptake of new diagnostic technologies in two developing country settings (Bangladesh and Uganda).

Results Diagnostic testing during ANC was generally viewed positively. Findings suggest that women and their families would prefer any proposed diagnostic tests be administered by skilled and trained providers. Communities expressed reservations in providing biological specimens during labour and the immediate postpartum period with discomfort and pain during labour and postpartum confinement cited as reasons for these preferences. Blood and urine were deemed the most acceptable specimens, and vaginal swabs were generally not viewed favourably. While providers were familiar with the concept of diagnostic testing, there is limited use of diagnostics in maternal health care, with HIV testing in Uganda being a notable exception. While 